\title{
Mobility Entropy and Message Routing in Community-Structured Delay Tolerant Networks
}

\author{
Hideya Ochiai \\ The University of Tokyo \\ jo2lxq@hongo.wide.ad.jp
}

\author{
Hiroshi Esaki \\ The University of Tokyo \\ hiroshi@wide.ad.jp
}

\begin{abstract}
Many message routing schemes have been proposed in the context of delay tolerant networks (DTN) and intermittently connected mobile networks (ICMN). Those routing schemes are tested on specific environments that involve particular mobility complexity whether they are random-based or sociologically organized. We, in this paper, propose community structured environment (CSE) and mobility entropy to discuss the effect of node mobility complexity on message routing performance. We also propose potential-based entropy adaptive routing (PEAR) that adaptively carries messages over the change of mobility entropy. According to our simulation, PEAR has achieved high delivery rate on wide range of mobility entropy, while link-state routing has worked well only at small entropy scenarios and controlled replicationbased routing only at large entropy environments.
\end{abstract}

\section{Categories and Subject Descriptors}

C.2.1 [Network Architecture and Design]: Wireless Comunication; C.2.2 [Network Protocols]: Routing Protocols

\section{General Terms}

Algorithms, Performance, Theory

\section{Keywords}

Delay Tolerant Networks, Mobility Entropy, CommunityStructured Environment, Potential-Based Routing

\section{INTRODUCTION}

The communication paradigm of delay and disruption tolerant networks (DTN)[9], such as hop-by-hop appli-

Permission to make digital or hard copies of all or part of this work for personal or classroom use is granted without fee provided that copies are not made or distributed for profit or commercial advantage and that copies bear this notice and the full citation on the first page. To copy otherwise, to republish, to post on servers or to redistribute to lists, requires prior specific permission and/or a fee.

AINTEC'08, November 18-20, 2008, Bangkok, Thailand.

Copyright 2008 ACM 978-1-60558-127-9/08/11 ...\$5.00. cation message delivery, is promising in many fields especially where stable Internet connectivity is not available (e.g., communication in disaster-affected areas, developing regions, wildlife tracking and wireless sensor networks). It has also been discussed in the context of intermittently connected mobile networks (ICMN), where an end-to-end connected path rarely or never exists because of highly dynamic properties of topology changes and node mobility.

Researchers have proposed several routing schemes for DTNs often assuming particular mobility environments. DTLSR $[8]$ has adopted link-state routing for the communication among villages in developing regions. MaxProp[4] and RAPID[1] discussed message delivery among city buses. PROPHET[18] and SOLAR[12] focused on particular sociological mobile scenarios and evaluated their proposed routing schemes on them. Random waypoint mobility(RWP) has been widely used for evaluation of message routing in $\operatorname{ICMNs}[25,22,14,3]$. These works commonly focus on their particular environments with regard to mobility complexity to discuss their proposed routing schemes.

We propose community-structured environment (CSE) for evaluating routing schemes over wide range of mobility complexity. In order to parameterize the complexity, we define mobility entropy in CSE. Mobility entropy works as an objective criterion of complexity. Small entropy is associated with well-structured mobile environments. A random or chaotic mobility model gives large entropy.

In this paper, we also propose potential-based entropy adaptive routing (PEAR) that carries out message routing adaptively over the change of mobility entropy. PEAR dynamically changes message replication level depending on mobility entropy to always achieve high delivery rate. A node basically transfers messages toward the nodes of higher delivery probability with less message replication at smaller entropy, but it replicates more messages at larger entropy to maintain delivery rate. PEAR is not aware of entropy by itself. Node mobility initiates replication, and this makes PEAR entropy-adaptive. 
PEAR inherits the concept of potential-based routing (PBR)[2], which is a family of message routing protocols that a node has a scalar value called potential for each destination and forwards messages toward the neighbor that has the lowest potential. The advantage of PBR is that a node can make forwarding decisions without a global knowledge of network topology. PBR only requires neighbor information for this purpose.

The forwarding decisions in PBR are made by potentials over nodes, which we call potential-field. We define a recurrence formula for potential-field computation in PEAR, which basically works in autonomously and totally distributed manner. The recurrence formulation enables dynamic computation of potential-field without using a global knowledge of network status. It only uses neighbor network status, but constructs global potential-fields appropriately.

We carry out simulations on various CSEs with changing mobility entropy in order that we investigate the effect of mobility entropy on message routing performance. We recognize that the performance is affected by many environmental features such as network bandwidth, media-access control (MAC) protocol, message buffer capacity as well as mobility. However, in this work, we assume an ideal environment (e.g., infinite network bandwidth and huge capacity of storage) to demonstrate the relationship between mobility entropy and routing performance.

This paper is organized as follows. In section 2, we present related works. In section 3 , we propose community structured environment. In section 4 , we define message delivery in PEAR. We propose a potential-field construction method in section 5 . We describe performance evaluation in section 6 . In section 7 , we discuss the evaluation result. In section 8 , we give the summary of this paper.

\section{RELATED WORK}

Traditionally, in order to evaluate the performance of routing in ICMNs, random-based mobility models have been adopted. Such mobility models include random waypoint(RWP), random walk(RW) and random direction(RD)[16]. It has been recently widely acknowledged that random-based mobility is unrealistic and that routing schemes are frequently discussed on sociologicallyorganized mobility models, which are studied in the context of buses[26], taxi[21], sociological orbit[11, 12] and pedestrians[5]. Community-based mobility is also proposed by [23], but it is basically random direction mobility which hierarchically defined. The problem is that these mobility models possess a particular environmental feature with regard to mobility entropy; e.g., random-based mobility gives extremely large entropy and sociologically-organized mobility gives smaller entropy. Community-structured environment, we intro- duced in this paper, enable seamless shifts among several mobility models in terms of entropy.

CSE is motivated by the work of Natasa et. al.[21] and SOLAR[12]. Natasa et. al. have analyzed approximately 800 taxi traces over three months and found stable concentration points $(\mathrm{CPs})$ where nodes encounter. SOLAR has proposed partially repetitive orbital mobility pattern that a node goes around in a small set of location points, which seems to be better suited to practical scenarios than random-based mobility. A CP corresponds to a community in CSE and node moves in a small set of communities in CSE. In this paper, we add the concept of mobility entropy in the context of CSE.

As for routing in DTNs and ICMNs, several routing schemes have been proposed. Link-state routing scheme was adopted to communication between villages in developing regions $[15,8]$. Depending on the methods of computing the link cost, maximum delivery probability(MDP), minimum expected delay (MED) and minimum expected dependent delay (MEDD) are proposed [6]. Basically, link-state routing is effective only in the case of well-structured (i.e., low-entropy) environment. Message path becomes meaningless at a highly dynamic mobile networks.

Epidemic routing [25] ensures message delivery even in partitioned networks of highly dynamic topology. Basically, epidemic routing is flooding-based routing scheme, which copies message to all the nodes encountered, and the copy-received nodes start to copy the message in the same manner. It ideally achieves minimum delivery latency, but, it is said that epidemic routing consumes lots of network resources and buffer space, which results in traffic congestion and poor performance in realistic scenarios.

Compared to epidemic routing, Spray and Wait[22] improves the overhead of message replication by controlling the maximum number of message copy. Message routing in Spray and Wait is composed of two phases. At first, in spray phase, the message source node makes message copies to neighbor nodes encounterd with limitation. Then, it waits until one of the nodes encounters the destination. Controlled replicationbased routing like Spray and Wait is useful only in the case of randomly contactable scenarios where random mobility guarantees delivery probability.

Potential-based routing (PBR) was proposed by Basu et. al. [2] in the context of traffic engineering in stable networks. In PBR, a node forward messages toward the neighbor that has the lowest potential. Followed by this work, PWave[19] has applied PBR to wireless sensor networks for routing of sensor readings to sink nodes. Volcano routing scheme (VRS)[10] is also an extention of PBR that computes potential-field to diffuse messages from densely message buffered areas. 

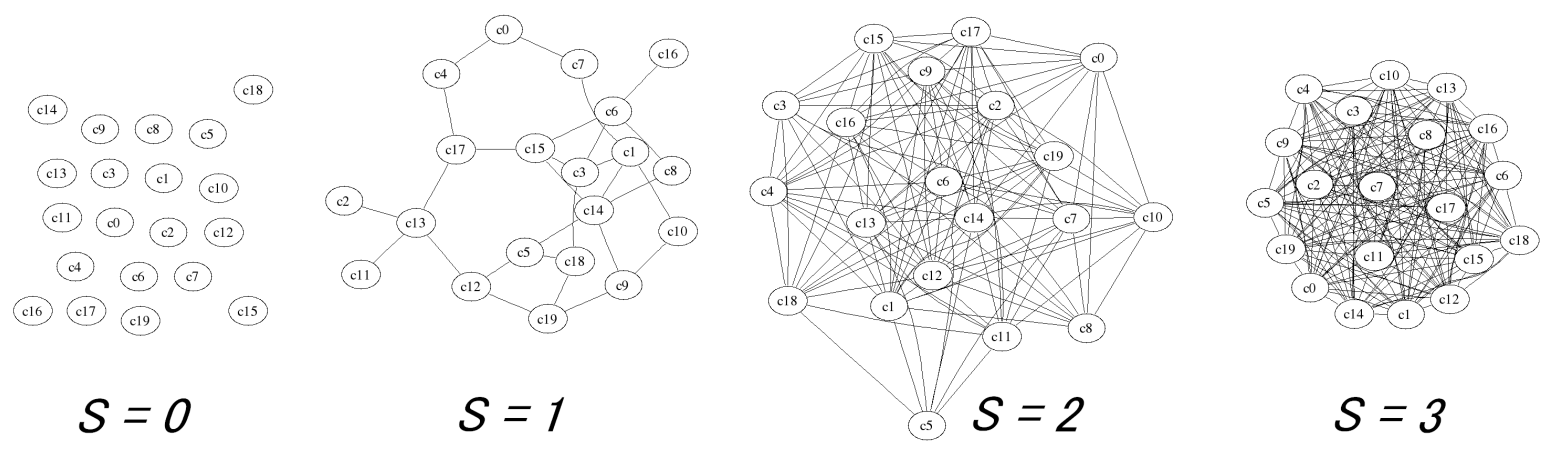

Figure 1: Communities Organized by Node Traces at Mobility Entropy $S=0,1,2,3$
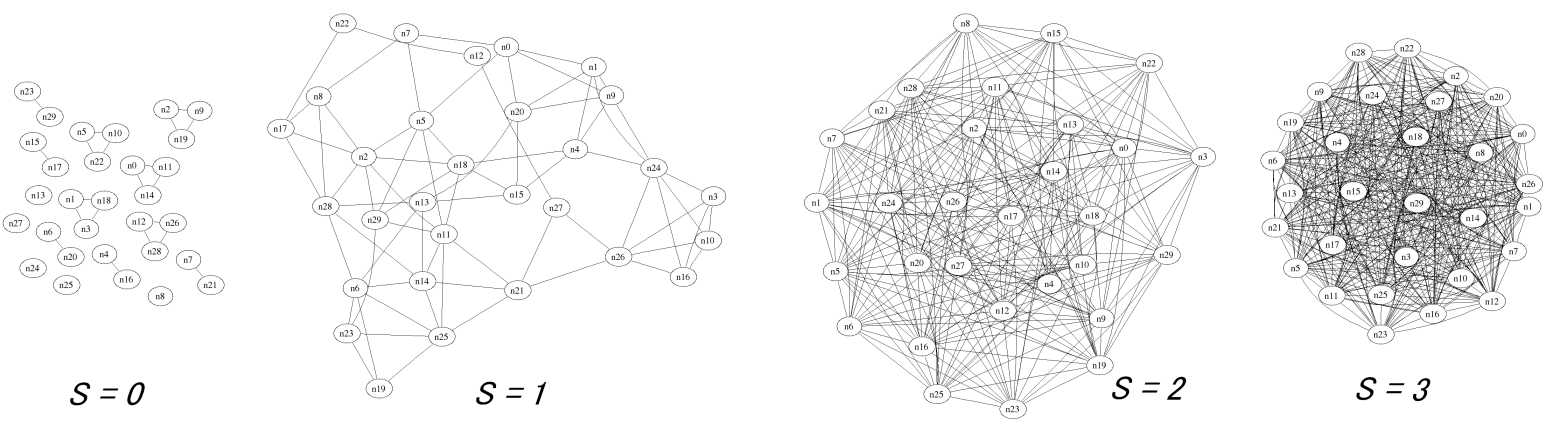

Figure 2: Nodes Organized by Contacts at Mobility Entropy $S=0,1,2,3$

Utility-based routing was proposed by [7] in mobile ad hoc networks (MANET) to support disconnected transitive communication. Utility is a scalar value that shows logical proximity to the destination. In that, utilitybased routing is the same as potential-based routing in nature. The node of the highest utility will relay message to its destination with higher probability than any other nodes. There are a number of utility computation methods in literature. History-based protocol was proposed by ZebraNet [17]. Chen et. al. [7] proposed Most-recently-noticed (MRN), Most-frequently-noticed (MFC). Spyropoulos et. al. proposed last-seen-first (LSF), most-mobile-first (MMF) and most-social-first (MSF) [24]. CAR[20] provides attribute-based general framework for computing utility that can be aware of remaining battery and other properties as well as contact statistics.

In this paper, we discuss entropy-adaptability in the context of potential-based routing. To our knowledge, entropy-adaptability has not been discussed in the previous works.

\section{COMMUNITY-STRUCTURED ENVIRON- MENT}

In order to describe mobile environments in terms of mobility entropy, we propose community-structured
environment(CSE).

Let $N$ be a set of nodes in the network, and $C$ a set of communities. A node $n \in N$ belongs to a sub set of $C$, which we denote by $C_{n}$. In community-structured environment, we define two mobility status: stay and transition. In the stay mode, a node $n$ stays at one of $C_{n}$, which is given by location $(n)$. In the transition mode, $n$ moves from community $c_{i}$ to community $c_{j}$ where $c_{i}, c_{j} \in C_{n}$ and $i \neq j$. A node is in contact with the nodes that stay in the same community. That is,

"Node $n$ and $k$ are within direct transmission range" $\Leftrightarrow$

"Node $n$ and $k$ are in contact with each other" $\Leftrightarrow$

$$
\exists c \in C, \operatorname{location}(n)=c \wedge \operatorname{location}(k)=c
$$

location $(n)$ gives undefined when node $n$ is in transition state.

We define CSE node mobility as follows $\left(\left\|C_{n}\right\|\right.$ gives the number of elements in Set $C_{n}$ ),

1. Node $n$ stays at community $c_{i} \in C_{n}$.

2. Choose a random value $r$ uniformly in $[0,1)$.

3. If $p<r$ or $\left\|C_{n}\right\|=1$, goto 1 . Parameter $p$ is probability of transition from stay mode to transit mode. 
4. Choose a destination community $c_{d}$ from $C_{n}-\left\{c_{i}\right\}$ at random.

5. Let $n$ move to $c_{d}$ with transitive time $T\left(c_{i}, c_{d}\right)$.

6. After $n$ reached the destination, $c_{i}:=c_{d}$ and goto 1.

We formally define mobility entropy $S$ of CSE as,

$$
S=\frac{1}{\|N\|} \sum_{n \in N} \log _{2}\left\|C_{n}\right\|
$$

Here, if every node belongs to the same number of communities(i.e., $\left.\left\|C_{i}\right\|=\left\|C_{j}\right\|\right), S$ can be described as,

$$
S=\log _{2} \Omega
$$

$\Omega$ is the number of communities that every node belongs to.

We use the above $S$ and $\Omega$ as a feature of CSE in the following discussion.

We show CSE instances in the case of $S=0,1,2,3$ in figure 1 and 2 . In figure 1 , a community is denoted by a vertex of the graph, and node traces are denoted by the edges. In figure 2 , a node is denote by a vertex of the graph, and a node-to-node contactability is denoted by an edge. A pair of vertexes that connected by an edge indicates that those nodes are possible to encounter with each other.

As these figures indicate, randomly contactable environment is characterized by larger entropy (e.g., $S=3$ ). Stable or well-structured mobile environments provide small entropy (e.g., $S=0,1$ ). In fact, random waypoint mobility[16] is given by setting $\Omega=\|C\|$, which is the case at the largest $S$. Village-to-village scenarios [8] could be categorized by small entropy.

\section{MESSAGE DELIVERY IN PEAR}

In this paper, we propose potential-based entropy adaptive routing (PEAR). Here, we focus on message delivery method under the assumption that potentialfields are already given. We discuss autonomous constrution of potential-fields in PEAR in the next section.

In the following discussion, we denote the neighbor nodes (including itself) of node $n$ by $n b r(n) . n b r(n)$ is a set of nodes within the same community of location $(n)$ if it is defined(i.e., in stay mode). Otherwise, $n b r(n)=$ $\{n\}$.

In potential-based routing(PBR)[2], a node has a scalar value that shows a kind of distance to its destination. We call the value potential and describe it as $V^{d}(n)$. When we consider the change of potential over time, we describe it as $V^{d}(n, t)$, which means the potential for destination $d$ at node $n$ at time $t$. In this section, we assume that $V^{d}(n)$ is given and we focus on message delivery on it. Computation of $V^{d}(n)$ is discussed in section 5 .
Basically, message delivery in PBR is carried out by forwarding messages toward the node of the lowest potential among its neighbors. After a node forwarded a message to the next node, it usually removes the message from the local buffer. In stable networks (i.e., wired and connected networks), this message delivery scheme is appropriate. However, in DTN scenarios, message delivery should be carried out more redundantly to improve delivery probability and latency.

In DTN environment, we consider that messages should not be just forwarded to the next node; it should be copied but should not be deleted from the local buffer. The copy-source node tries to make another copy of messages again when it encounters to another node. Message replication in this way will improve the delivery probability and latency. When we introduce replication, the network must deal with replica management that involves message deletion after it has reached the destination.

In this context, we distinguish the terms of copy, forward, replicated messages and delete as,

Copy: the process of making a clone of a message from this node into the other node.

Forward: the process of making a clone of a message from this node into the other node and deleting the original message.

Replicated messages: messages left in the network by the process of copy.

Delete: the process of eliminating replicated messages from the network.

\subsection{Selection of Next Hop Nodes}

Let $M$ be a set of messages in the network, and $M^{d}(\subset$ $M$ ) be the messages which destination is $d$. To deliver a message $m \in M^{d}$, node $n$ must determine the next hop nodes of $m$, at first. We define two next hop selection schemes: i.e., best or single candidate selection(BCS) and multiple candidate selection(MCS).

Best (or Single) Candidate Selection (BCS):

$$
\begin{aligned}
n \operatorname{exthop}_{B C S}^{d}(n) & = \\
\{k \mid k \in \operatorname{nbr}(n) & \wedge F_{k}^{d}(n)=\max _{j \in \operatorname{nbr}(n)}\left\{F_{j}^{d}(n)\right\}>\alpha(M)
\end{aligned}
$$

Here, $F_{k}^{d}(n)$ is the force that affects on the message $m^{d}$ from node $n$ toward neighbor $k$, which we define as

$$
F_{k}^{d}(n)=V^{d}(n)-V^{d}(k)
$$

Lower potential of neighbor $k$ enlarge the force from node $n$ to $k$.

In BCS, node $n$ chooses the neighbor $k$ that gives the maximum $F_{k}^{d}(n)$ as the next hop of $M^{d}$ at every time unit. Here, the force must be more than a constant 


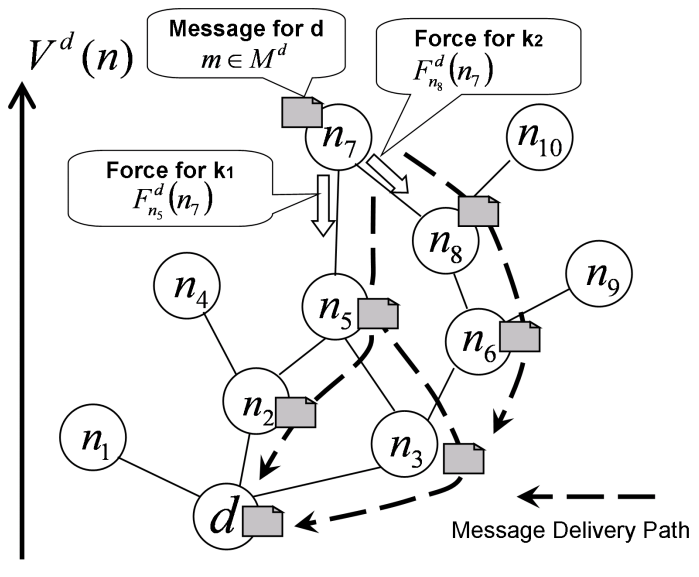

Figure 3: Message Delivery in PEAR

value $\alpha$, the threshold of the least force level. Otherwise, no selection are made for destination $d$. Nodes encounter and leave as time elapses, and the best candidate changes according to $n b r(n)$.

Multiple Candidate Selection (MCS):

$\operatorname{nexthop}_{M C S}^{d}(n)=\left\{k \mid k \in n b r(n) \wedge F_{k}^{d}(n)>\beta\right\}$

In MCS, next hop nodes are such neighbors that the force is more than $\beta(>0)$. MCS chooses multiple next hop nodes at the same time.

Figure 3 demonstrates how PEAR delivers a message $m\left(\in M^{d}\right)$ to destination $d$. In this figure, nodes are mapped according to their potential $V^{d}(n)$ in the vertical axis, and the edges show node-to-node contactability (i.e., intermittent connectivity) between nodes. At first, $m$ is possessed by $n_{7}$, which has the highest potential in the figure. During $n_{7}$ is not connected to neither of $n_{5}$ or $n_{8}$, it does nothing. When it encounters both of $n_{5}$ and $n_{8}$ at the same time, it copies the message to $n_{5}$ since $F_{n_{5}}^{d}\left(n_{7}\right)>F_{n_{8}}^{d}\left(n_{7}\right)$ in BCS, whereas in MCS it copies them to both of $n_{5}$ and $n_{8}$. In BCS ( $n_{8}$ does not possess $m$ ), after $n_{5}$ has left from $n_{7}, n_{7}$ copies $m$ to $n_{8}$ since $F_{n_{8}}^{d}\left(n_{7}\right)$ now provides the strongest force among its neighbors. $n_{5}, n_{8}$ and any other nodes behave in the same way and the message $m$ will be copied to the lower potential nodes until they reach the destination.

At small entropy (e.g., $S=1$ ), PEAR only uses a small set of nodes for message delivery, saving resources as much as possible. As entropy $S$ becomes larger, probability of meeting of nodes (e.g., between $n_{5}$ and $n_{7}$ ) decreases and message delivery on that paths may fail. However, PEAR maintains delivery probability even in larger $S$ by replicating more messages in the network. At large entropy, $n_{7}$ also has links with $n_{1}, n_{4}$ and $n_{9}$. Thus, $m$ will be copied to those nodes, which increases the replication level, and achieves high delivery rate.

\subsection{Replica Management}

After nexthop ${ }^{d}(n)$ is determined by BCS or MCS at node $n$. $n$ tries to copy message $m \in M^{d}$ to them. Here, some of them may already have a replica of the message or others may know that the message has already reached the destination. Replica management should be carried out in PEAR in order to reduce the overhead of message duplication and to efficiently use buffer space by removing replicas of delivered message from the network.

In PEAR, message $m$ must contain the following information as well as the message body in its header at least.

- MessageID

- Destination

- Time to Live (TTL)

The following information should be managed at every node locally for each $m$.

- DisseminationTTL

- IsDelivered

MessageID must be uniquely defined in the network. TTL is a message life time which decreases, for example, every second. When TTL reaches zero, the message expires (with freeing memory allocated for $m$ including headers). TTL corresponds to the left time for delivery deadline.

DisseminationTTL describes whether node $n$ can send the message to other nodes or not. Initially, node $n$ sets it to TTL of the message when received the message. It decreases in the same manner as TTL does. As we describe later, DisseminationTTL changes depending on the process of message replication. If it has expired, node $n$ does not transfer the message to its next hop nodes any longer and deletes the body of it (with freeing allocated memory space for message body), but continues to check the existence of a delivery certification at the next hop nodes. IsDelivered shows whether delivery has been certified or not. Originally, when this node receives a message, IsDelivered is set to false. After finding the delivery certification, it is set to true. The first certificate will be published by the destination node after it has received the message.

Of course, in practice, the message source identifier should also be included in the message header along with the destination. However, PEAR does not care about the source of the message, so we consider it as optional.

We define message delivery and replica management algorithm on given nexthop ${ }^{d}$ and $m \in M^{d}$ in figure 4 .

In the algorithm, check_message_status $(k, m)$ tries to find node $k$ and asks about the status of message $m$ and replies one of 


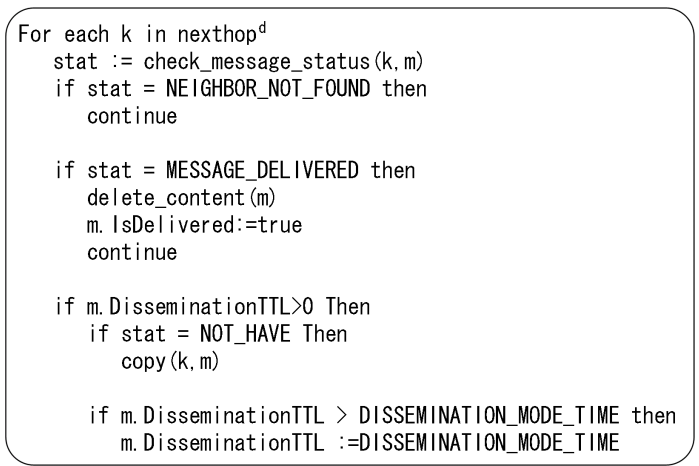

Figure 4: Algorithm of Message Delivery and Replica Management

\section{- NEIGHBOR_NOT_FOUND}

- MESSAGE_NOT_HAVE

- MESSAGE_ALREADY_HAVE

\section{- MESSAGE_DELIVERED}

transfer $(k, m)$ makes a copy of message $m$ into $k$. delete_content $(m)$ removes the body of $m$ and frees the allocated memory.

\subsection{Loop-Freeness}

Loop-freeness in potential-based routing is proved by [2] in the case of static potential-field. Basically, a message which has been forwarded to the lower potential node cannot come again from the upper potential node.

In our modified version of PBR, message delivery is carried out by copying, not by forwarding. Message remains at the nodes where it has infected. Therefore, even in dynamically potential-changing scenarios, messages never loop in PEAR.

\section{POTENTIAL-FIELD CONSTRUCTION}

In the previous section, we described a message delivery scheme in PEAR on a given potential-field. In this section, we describe potential-field construction method in PEAR, which autonomously and dynamically computes the field. Potential computation in PEAR does not require global topology information. It only uses neighbor information but makes an appropriate potential field globally. This is the same property that next hop decision schemes possess.

We propose a recurrence formula that computes the field which has the above feature in this section.

\subsection{Recurrence Formulation}

The recurrence formula we propose for potential-field construction is,

$$
\begin{aligned}
V^{d}(n, t+1) & =V^{d}(n, t) \\
& +D \min _{k \in n b r(n)}\left\{V^{d}(k, t)-V^{d}(n, t)\right\} \\
& +\rho \\
V^{d}(n, 0) & =0 \\
V^{n}(n, t) & =0 \\
0<\rho<D & , \quad 0<D<1
\end{aligned}
$$

The potential of node $n$ at the next time step $(t+1)$ is calculated on the potential of neighbors at current time $t$. Basically, it inflates by $\rho$, but the inflation is depressed by the smallest potential among neighbors. This depression is weighted by $D$, which we call minimum-potential diffusion constant.

Eqn. 8 gives the initial condition; i.e., all the potential value starts at zero. Eqn. 9 gives a boundary condition that a node must always have zero potential for itself, which means that the potential at the destination must be always set to zero.

The potential at the destination node $V^{d}(d)=0$ diffuses from the message destination around the network with some increase. In this way, nodes farther from the destination gets higher potential, and nodes closer to the destination gets lower potential. Message delivery is carried out from the higher potential node to the lower potential node.

\subsection{Dynamics}

In figure 5, we illustrate how PEAR autonomously constructs a potential-field by Eqn. 7 and achieves message delivery. We assume a quite simple case to make the discussion easy: i.e., four nodes and three communities. Here, $n_{1}$ belongs to a community $c_{1} \cdot n_{2}$ belongs to $c_{1}$ and $c_{2} . n_{3}$ belongs to $c_{2}$ and $c_{3}$, and $n_{4}$ to $c_{3}$. Nodes are in contact with each other only when they are located at the same community; i.e., $n_{1}$ and $n_{2}$ are in contact when $n_{2}$ locates at $c_{1}, n_{2}$ and $n_{3}$ are in contact when they stay at $c_{2}$, and so on. A contact is denoted by a line, and disconnection is denoted by dashed lines. The vertical axis shows potential for destination $n_{1}$.

(a) Initially, nodes have the same potential value at zero. They except $V^{n_{1}}\left(n_{1}\right)$ start to increase by $\rho$.

(b) As time elapses, $V^{n_{1}}\left(n_{2}\right)$ stays at $\frac{\rho}{D}$, while $V^{n_{1}}\left(n_{3}\right)$ and $V^{n_{1}}\left(n_{4}\right)$ continues to increase at speed $\rho$. At this time, $n_{2}$ can send its $M^{n_{1}}$ to $n_{1}$, while $n_{3}$ and $n_{4}$ store them in the local memory.

(c) The physical topology has changed, and only $n_{2}$ and $n_{3}$ are in contact. $V^{n_{1}}\left(n_{2}\right)$ starts to increase at speed $\rho$, while $V^{n_{1}}\left(n_{3}\right)$ decreases toward $V^{n_{1}}\left(n_{2}\right)$. Here, $n_{3}$ copies its $M^{n_{1}}$ to $n_{2}$.

(d) When the link between $n_{2}$ and $n_{3}$ is disrupted and instead the link between $n_{3}$ and $n_{4}$ has set up, 


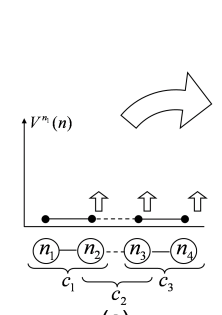

(a)

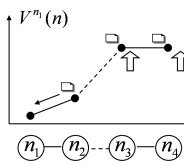

(b)
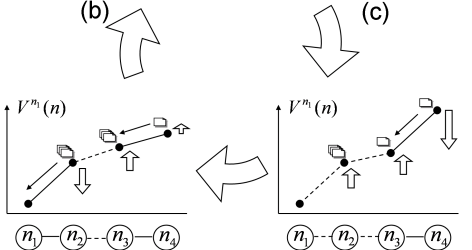

(e)

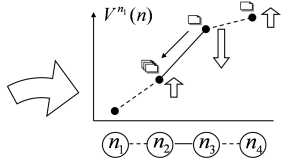

$\int^{(c)}$

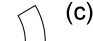

(d)
Figure 5: Dynamics of Potential-Field Construction over the Change of Node-to-Node Connectivity

$V^{n_{1}}\left(n_{2}\right)$ and $V^{n_{1}}\left(n_{3}\right)$ increases at speed $\rho$, while $V^{n_{1}}\left(n_{4}\right)$ decreases toward $V^{n_{1}}\left(n_{3}\right)$. In this situation, $n_{4}$ copies its $M^{n_{1}}$ to $n_{3}$.

(e) $n_{2}$ is now in contact with $n_{1} \cdot V^{n_{1}}\left(n_{2}\right)$ decreases to $\frac{\rho}{D}$, and $n_{2}$ transfer its $M^{n_{1}}$ to $n_{1}$.

If the network topology do not change, (e) goes to (b). In this way, potential-field is autonomously constructed and maintained so that messages could be delivered to the destination.

\subsection{Performance Study}

We discuss the performance of potential-field construction in PEAR with regard to computation and transmission cost.

In order to inform potentials to neighbors, we assume that a node periodically advertises potentials that it has (e.g., every ten second). This advertisement from $n$ contains potentials bound for all the destinations (i.e., $\left.\left\{V^{d}(n) \mid \forall d \in N\right\}\right)$. Thus, the size of advertisement is given by $O(m)$ where $m$ is the number of elements in $N$ (i.e., $m=\|N\|)$.

The computation of the next potential value (Eqn. 7) can be also performed at $O(m)$ by using sorted array. Suppose that $A$ is a sorted array which members are denoted by $\left\{a_{1}, a_{2}, \ldots a_{j}\right\}$. Here $a_{1}, \ldots, a_{j}$ is sorted into increasing order. For each destination, by maintaining potentials of neighbors by $A, a_{1}$ always gives the minimum potential value among its neighbors. Therefore, Eqn. 7 can be calculated at $O(1)$. Since the potential must be calculated in parallel for each destination, the total computation cost is $O(m)$. As for the total maintenance cost of $A$ (i.e., the cost of inserting, updating, and deleting potential values), it carries out at $O(h \mathrm{~m})$, where $h$ denote the number of the neighbors(i.e., $h=\|n b r(n)\|)$.

\section{SIMULATION}

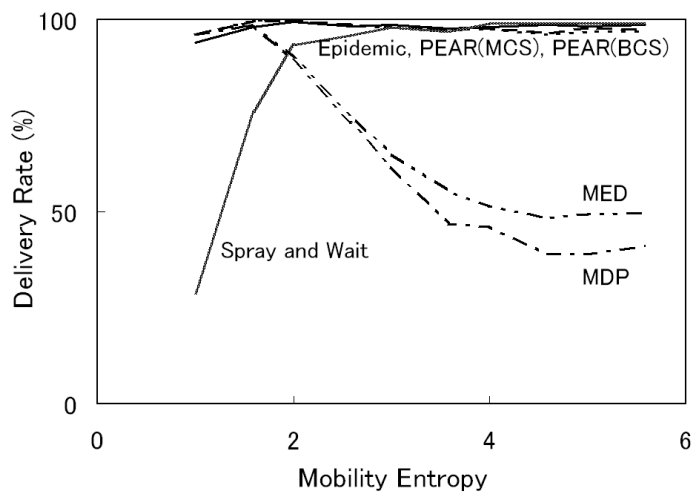

Figure 6: Message Delivery Rate

We evaluated PEAR, regarding to delivery rate and total message transmissions, on various CSEs by simulation. The purpose of this experiment is to analyze the features of routing schemes in terms of mobility entropy. Thus, we carried out the simulation without being aware of transmission properties (e.g., node-to-node link bandwidth and average message size). In this way, we focused on an ideal case where the effect of them can be ignored.

We set 100 nodes over 50 communities throughout the simulation with changing $\Omega$ from 2 to 48: i.e., entropy $S$ from 1 to 5.6 . We assumed the case that every node belongs to the same number $(=\Omega)$ of communities in each CSE.

Throughout the experiment, we set $D=0.001$ and $\rho=0.00001$ for potential-field construction (Eqn. 10), and $\alpha=0.8$ and $\beta=0.8$ for next hop selection (Eqn. 4 and Eqn. 6). The message lifetime was set to 20000.

We carried out the simulation of node mobility, potential field construction and message delivery during the time interval $[-50000,20000]$. While $t \in[-50000,0)$, no messages were submitted into the network; only the movement of nodes and potential-field construction were simulated. At $t=0$, every node sent messages to all the nodes in the network. While $t \in(0,20000]$, message delivery was also simulated as well as the node movement and potential-field construction.

We evaluated PEAR with other routing schemes such as epidemic routing[25], Spray and Wait (2-hop scheme) [22], Minimum Expected Delay(MED) and Maximum Delivery Probability(MDP)[6]. In the comparison with these schemes we have prepared completely the same set of CSEs.

\subsection{Delivery Rate}

Figure 6 shows the relationship between message delivery rate and mobility entropy.

Link-state routing (i.e., MED and MDP) achieved 
about $95 \%$ message delivery at $S=1$, but it failed $50 \%$ at larger entropy. Spray and Wait delivered only $28 \%$ of messages at $S=1$, whereas it delivered about $95 \%$ at larger entropy. PEAR achieved more than $95 \%$ delivery rate over any entropy environments, which is almost the same level that Epidemic routing did. These results indicate that PEAR has dynamically adapted to any given environments whether they are highly-dynamic or relatively well-organized. However, link-state routing and Spray and Wait has achieved good performance at the specific situations.

Figure 7 shows the change of delivery rate during time $[0,20000]$ at mobility entropy $S=1,3,5 . \quad S=1,3,5$ corresponds to $\Omega=2,8$ and 32 (Eqn. 3). From these graphs we could read that PEAR, MED and MDP at larger entropy delivered messages slower than those at smaller entropy. However, PEAR showed good tolerance of delivery latency against the increase of mobility entropy compared to MED and MDP.

The latency of message delivery in MED and MDP gets large sharply as $S$ increases, resulting in unsuccessful message delivery at time 20000. As for other routing schemes, Epidemic routing totally performed a good performance with regard to delivery rate, and Spray and Wait stopped the increase of message delivery rate at $28 \%$ around time 5000 at $S=1$, but the rate sharply increased at $S=3,5$.

From these results, we summarize that PEAR is useful for wider mobility entropy scenarios than the other routing schemes except Epidemic routing. Link-state routing (i.e., MED and MDP) is just useful at quite small entropy. Spray and Wait routing is useful for larger entropy scenarios, where nodes are possible to directly contact with most of the nodes in the network.

\subsection{Total Message Transmissions}

Total message transmissions is the total count of application message exchange among nodes. Figure 8 shows the relationship between total message transmissions and entropy. PEAR(BCS) reduced the transmissions to about $11 \%$ (at $S=1$ ) and $23 \%$ (at $S=5$ ) of Epidemic routing. Link-state routing (i.e., MED and MDP) transmitted about 3.5\% of Epidemic routing at $S=1$, where they achieved high delivery rate. Spray and Wait transmitted about $12 \%$ at $S=5$. PEAR generated two or three times more transmissions than link-state routing and Spray and Wait.

Figure 9 shows total message transmissions over the time interval from $t=0$ to $t=20000$. These graphs show the summary of transmission: e.g., 1000 transmissions at time 3000 means that 1000 messages have been exchanged in the network during $[0,3000]$. From the results we read that message delivery in Spray and Wait was carried out mostly during $[0,5000]$ at any CSEs. This is the same feature of Epidemic routing. Another

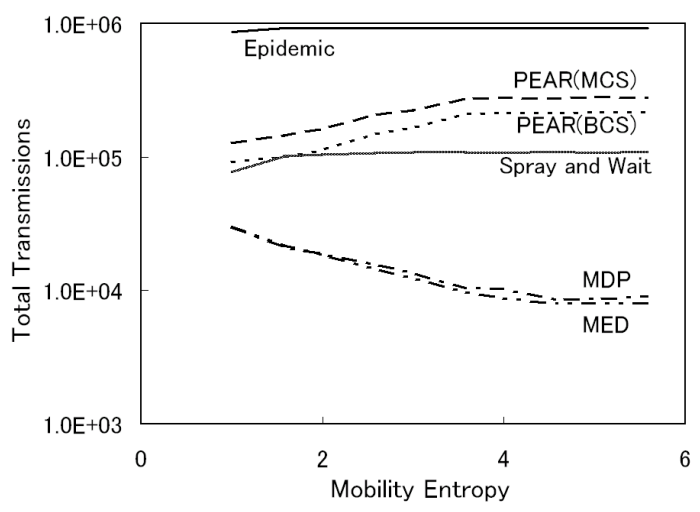

Figure 8: Total Message Transmissions

thing we read from the results is that link-state routing and PEAR carried out the delivery process gradually at $S=3,5$ though it has finished around $t=5000$ at $S=1$.

\section{DISCUSSION}

Introduction of community-structured environment (CSE) has been successful in that we could seamlessly classify mobile environments and analyze routing performance on the environments. By changing the entropy of CSEs, we could clearly read the features of routing schemes. Link-state routing (i.e., MDP and MED) has performed well only at smaller entropy cases such as village-to-village communications. Spray and Wait has worked well at larger entropy, where nodes can randomly contact with each other. PEAR, which we have proposed in this paper, has achieved high delivery rate over any entropy scenarios with transmission reduction to approximately $10 \%$ to $20 \%$ compared to Epidemic routing.

At smaller entropy, PEAR has achieved high delivery rate by aggressively transferring a message using the potential-field developed from the relatively stable mobile environment. In this case, the selected next hop nodes are most capable of carrying messages to the closer nodes to the destination. However, Spray and Wait just makes a copy to the nodes around, thus it failed to deliver most of the messages in our experiment.

At larger entropy, PEAR has also achieved high delivery rate by making a lot of message replicas in the network. In such environments, we consider that routing scheme must shift from message transfer scheme to mobility-based scheme: i.e., utilization of mobility is the primary factor of successful message delivery. In that Spray and Wait was effective. PEAR is also effective because a node becomes to replicate messages as entropy becomes larger. However, link-state routing could not adapt to the large entropy environments because it sticks to transfer a message to the given next 

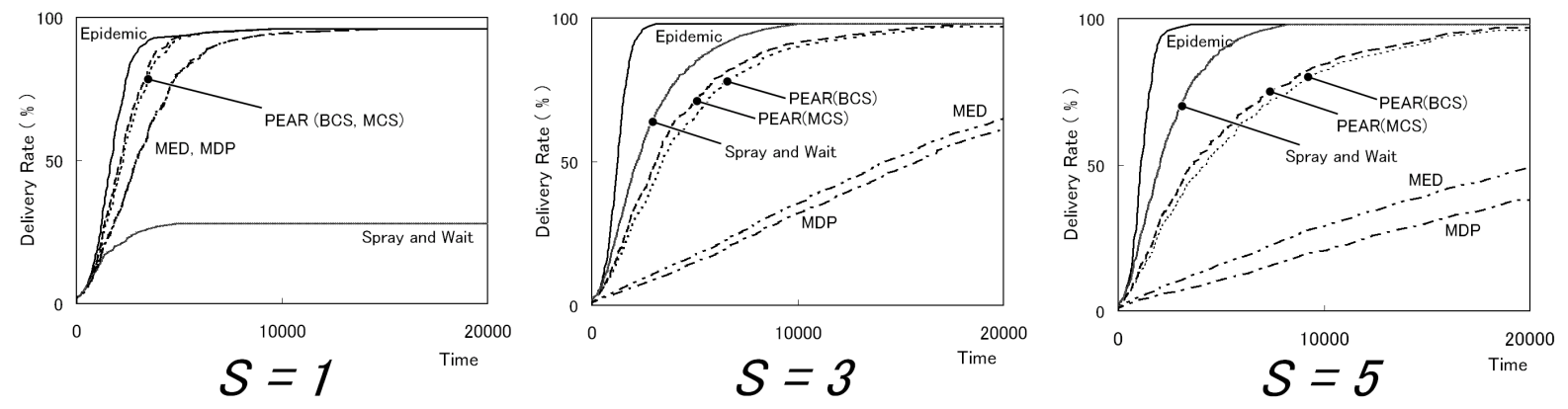

Figure 7: Delivery Rate at Entropy $S=1,3,5$
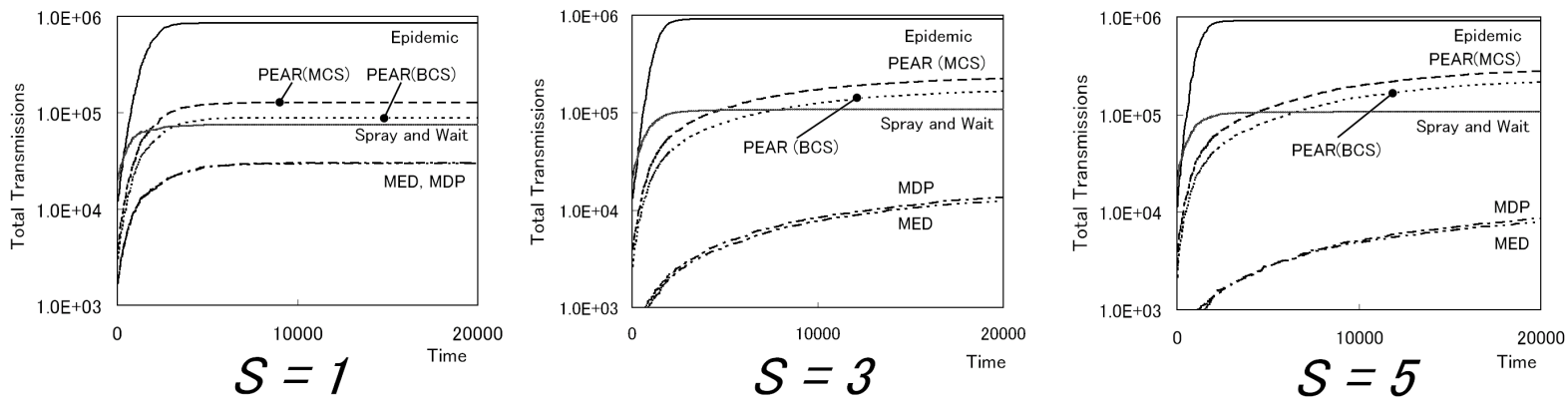

Figure 9: Total Message Transmissions at Entropy $S=1,3,5$

hop node.

According to our experiment, BCS and MCS showed almost the same feature with regard to delivery rate and total transmissions. MCS achieved almost the same delivery rate with about 1.4 times of transmissions in most cases. This result indicates that BCS scheme will be enough in practice.

The role of this paper was to analyze the properties of PEAR with regard to mobility entropy with comparison of other proposed routing schemes. For the next steps, we are considering about deployment into realistic scenarios. The actual performance will be also determined by other aspects of the environment besides mobility entropy such as radio bandwidth, media-access control (MAC) protocol, source-to-destination message traffic, buffer capacity and rate of failure. Epidemic routing could be suffered from congestion under limited bandwidth. According to [13], 10\% transmission reduction will improve the performance significantly in terms of congestion. Deployment of PEAR on various testbeds are required for further analysis and improvement in the near future.

\section{CONCLUSION}

We proposed community-structured environment(CSE) and potential-based entropy adaptive routing(PEAR) in this paper. CSE has enabled the classification of mobile environments in terms of mobility entropy. In CSE, stable or well-structured mobile environments are characterized by small entropy. Randomly contactable environments are characterized by large entropy.

Using CSE, we have analyzed the features of routing schemes by simulation. In our experiment, link-state routing (e.g., MED and MDP) has worked well at small entropy environments such as $S=1$ but failed to $50 \%$ delivery at larger entropy. Spray and Wait has achieved good performance at larger entropy, but only $28 \%$ messages have been delivered at $S=1$.

PEAR has achieved more than 95\% message delivery over any mobility entropy environments by adaptively changing the message delivery form. At small entropy, PEAR has aggressively transferred a message in hop-by-hop manner using the appropriately developed potential-fields. At large entropy, PEAR has automatically shifted to let mobility deliver the message with making more replicas in the network. In this way, PEAR has maintained the delivery rate.

\section{Acknowledgement}

This research was supported in part by national institute of information and communications technology (NICT), Japan.

\section{REFERENCES}


[1] A. Balasubramanian, B. N. Levine, and A. Venkataramani. DTN routing as a resource allocation problem. In ACM SIGCOMM, 2007.

[2] A. Basu, A. Lin, and S. Ramanathan. Routing using potentials: A dynamic traffic-aware routing algorithm. In ACM SIGCOMM 2003, 2003.

[3] J. Boice, J. J. Garcia-Luna-Aceves, and K. Obraczka. On-demand routing in disrupted environments. LNCS Networking, 4479:155-166, nov 2007.

[4] J. Burgess, B. Gallagher, D. Jensen, and B. N. Levine. MaxProp: Routing for vehicle-based disruption-tolerant networks. In IEEE INFOCOM, 2006.

[5] A. Chaintreau, P. Hui, J. Crowcroft, C. Diot, R. Gass, and J. Scott. Impact of human mobility on the design of opportunistic forwarding algorithms. In IEEE INFOCOM, 2006.

[6] C. Chen and Z. Chen. Evaluating contacts for routing in highly partitioned mobile networks. In ACM MobiOpp, 2007.

[7] X. Chen and A. L. Murphy. Enabling disconnected transitive communication in mobile ad hoc networks. In ACM POMC, 2001.

[8] D. Demmer and K. Fall. DTLSR: Delay tolerant routing for developing regions. In $A C M N S D R$, 2007.

[9] K. Fall. A delay-tolerant network architecture for challenged internets. In ACM SIGCOMM, 2003.

[10] Y. Ganjali and N. McKeown. Routing in a highly dynamic topology. In IEEE SECON, 2005.

[11] J. Ghosh, H. Q. Ngo, and C. Qiao. Mobility profile based routing within intermittently connected mobile ad hoc networks (ICMAN). In ACM IWCMC, 2006.

[12] J. Ghosh, S. J. Philip, and C. Qiao. Sociological orbit aware location approximation and routing (SOLAR) in MANET. ACM Ad Hoc Networks, 5(2):189-209, mar 2007.

[13] Z. Haas, J. Y. Halpern, and L. Li. Gossip-based ad hoc routing. In IEEE INFOCOM, 2002.

[14] J. Hahner, C. Becker, and K. Rothermel. A protocol for data dissemination in frequently partitioned mobile ad hoc networks. In IEEE ISCC, 2003.

[15] S. Jain, K. Fall, and R. Patra. Routing in a delay tolerant network. In ACM SIGCOMM, 2004.

[16] A. Jindai and K. Psounis. Fundamental mobility properties for realistic performance analysis of intermittently connected mobile networks. In IEEE Percom, 2007.

[17] P. Juang, H. Oki, Y. Wang, M. Martonosi, L.-S. Peh, and D. Rubenstein. Energy-efficient computing for wildlife tracking: Design tradeoffs and early experiences with ZebraNet. In $A C M$ SIGOPS, pages 96-107, 2002.

[18] A. Lindgren, A. Doria, and O. Schelen. Probabilistic routing in intermittently connected networks. LNCS, 3126:239-254, sep 2004.

[19] H. Liu, Z.-L. Zhang, J. Srivastava, and V. Firoiu. PWave: A multi-source multi-sink anycast routing framework for wireless sensor networks. LNCS Networking, 4479:179-190, nov 2007.

[20] M. Musolesi, S. Hailes, and C. Mascolo. Adaptive routing for intermittently connected mobile ad hoc networks. In IEEE WoWMoM, 2005.

[21] N. Sarafijanovic-Djukic, M. Piorkowski, and M. Grossglauser. Island Hopping: Efficient mobility-assised forwarding in partitioned networks. In IEEE SECON, 2006.

[22] T. Spyropoulos, K. Psounis, and C. S. Raghavendra. Spray and Wait: An efficient routing scheme for intermittently connected mobile networks. In ACM SIGCOMM, 2005.

[23] T. Spyropoulos, K. Psounis, and C. S. Raghavendra. Performance analysis of mobility-assised routing. In ACM MobiHoc, 2006.

[24] T. Spyropoulos, T. Turletti, and K. Obraczka. Utility-based message replication for intermittently connected heterogeneous networks. In IEEE WoWMoM, 2007.

[25] A. Vahdat and D. Becker. Epidemic routing for partially-connected ad hoc networks. Technical report, Duke University, 2000.

[26] X. Zhang, J. Kurose, B. N. Levine, D. Towsley, and H. Zhang. Study of a bus-based disruption-tolerant network: Mobility modeling and impact on routing. In ACM MobiCom, 2007. 